\title{
Desmin and dystrophin abnormalities in upper airway muscles of snorers and patients with sleep apnea
}

\author{
Farhan Shah', Karl A. Franklin², Thorbjörn Holmlund', Eva Levring Jäghagen ${ }^{4}$, Diana Berggren³, \\ Sture Forsgren ${ }^{1}$ and Per Stål ${ }^{{ }^{*}}$ (D)
}

\begin{abstract}
Background: The pathophysiology of obstruction and swallowing dysfunction in snores and sleep apnea patients remains unclear. Neuropathy and to some extent myopathy have been suggested as contributing causes. Recently we reported an absence and an abnormal isoform of two cytoskeletal proteins, desmin, and dystrophin, in upper airway muscles of healthy humans. These cytoskeletal proteins are considered vital for muscle function. We aimed to investigate for muscle cytoskeletal abnormalities in upper airways and its association with swallowing dysfunction and severity of sleep apnea.
\end{abstract}

Methods: Cytoskeletal proteins desmin and dystrophin were morphologically evaluated in the uvula muscle of 22 patients undergoing soft palate surgery due to snoring and sleep apnea and in 10 healthy controls. The muscles were analysed with immunohistochemical methods, and swallowing function was assessed using videoradiography.

Results: Desmin displayed a disorganized pattern in $21 \pm 13 \%$ of the muscle fibres in patients, while these fibers were not present in controls. Muscle fibres lacking desmin were present in both patients and controls, but the proportion was higher in patients ( $25 \pm 12 \%$ vs. $14 \pm 7 \%, p=0.009)$. The overall desmin abnormalities were significantly more frequent in patients than in controls ( $46 \pm 18 \%$ vs. $14 \pm 7 \%, p<0.001)$. In patients, the C-terminus of the dystrophin molecule was absent in $19 \pm 18 \%$ of the desmin-abnormal muscle fibres. Patients with swallowing dysfunction had 55 $\pm 10 \%$ desmin-abnormal muscle fibres vs. $22 \pm 6 \%$ in patients without swallowing dysfunction, $p=0.002$.

Conclusion: Cytoskeletal abnormalities in soft palate muscles most likely contribute to pharyngeal dysfunction in snorers and sleep apnea patients. Plausible causes for the presence of these abnormalities is traumatic snoring vibrations, tissue stretch or muscle overload.

Keywords: Muscle, Upper airway dysfunction, Dysphagia, Pathophysiology, Cytoskeletal abnormalities, desmin, dystrophin

\section{Background}

Obstructive sleep apnea is a highly prevalent disorder associated with adverse health consequences [1]. The disorder is characterized by repetitive narrowing and collapse of the upper airways, with snoring and subsequent hypoxia [2]. Inadequate dilating muscle forces are suggested as an important factor in the pathophysiology of obstructive sleep apnea. Nerve injuries due to traumatic snoring vibration have been proposed as one of the causes for the inability

\footnotetext{
* Correspondence: per.stal@umu.se

1 Department of Integrative Medical Biology, Laboratory of Muscle Biology, Umeå University, SE-901 87 Umeå, Sweden

Full list of author information is available at the end of the article
}

of the upper airway muscles to maintain patency during sleep and for the commonly occurring swallowing dysfunction in snorers and sleep apnea patients [3-14]. Although evidence of upper airway neuropathy exists in snorer and sleep apnea patients, acquired muscle injuries as a contributing cause for muscle weakness and pharyngeal dysfunction has gained less attention [11].

We have earlier reported that a small subpopulation of fibres in soft palate muscles of healthy humans lacked or had a truncated form of two cytoskeletal proteins, desmin, and dystrophin [15]. This is specifically interesting since these two cytoskeletal filaments are considered to be ubiquitous and vital for muscle function [16]. Desmin

(c) The Author(s). 2019 Open Access This article is distributed under the terms of the Creative Commons Attribution 4.0 International License (http://creativecommons.org/licenses/by/4.0/), which permits unrestricted use, distribution, and 
is the major intermediate filament (IF) in mature muscles [16]. It is located at the periphery of the Z-disc and links the entire contractile apparatus to the subsarcolemmal cytoskeleton, the cell nuclei and to other organelles such as mitochondria [17-19]. The network of desmin filaments supports the structural and mechanical integrity of the muscle cell during contraction and contributes to force transmission and load bearing [16]. Dystrophin is localized in the inner part of the sarcolemma where it binds other cytoskeletal protein filaments in the muscle cell to the surrounding extracellular matrix (ECM) through the cell membrane [20]. The $\mathrm{C}$-terminus domain of the dystrophin molecule is associated with the membrane-spanning dystrophin-associated protein complex (DAPC), whereas its $\mathrm{N}$-terminus interacts with actin filaments. The DAPC has significant roles in stabilizing sarcolemma and transmitting force generated in the muscle sarcomere to ECM [21]. In genetic myopathies and animal gene knockout experiments, the absence of these proteins leads to progressive muscle weakness $[22,23]$.

Presence of abnormalities in cytoskeletal proteins in upper-airway muscles of snoring and sleep apnea patients and its impact on pharyngeal function has not been investigated. Therefore, we aimed to investigate for desmin and dystrophin abnormalities in soft palate muscles of snoring and sleep apnea patients and to evaluate whether these abnormalities relate to deviations in swallowing function and severity of obstructive sleep apnea.

\section{Methods}

\section{Patients and controls}

Twenty-two consecutive patients ( 1 female, 21 males) referred for upper-airway surgery because of snoring and sleep apnea were included. The exclusion criteria were smoking, previous palatal surgery, systemic disease, medications, and drug abuse. The mean age was 45 years (range 29-60), and the mean body mass index (BMI) was $28 \mathrm{~kg} / \mathrm{m}^{2}$ (range 21-34). Ten voluntary controls, all males, mean age 38 years (range $30-51$ ) and mean BMI $24 \mathrm{~kg} / \mathrm{m}^{2}$ (range 22-30), were recruited through advertisements. The exclusion criteria were similar as in patients, but also included habitual snoring and sleep apnea. For reference, a biopsy from an arm muscle, biceps brachii, and a thigh muscle, vastus lateralis, were acquired from two healthy adult male subjects.

\section{Sleep apnea recordings}

All patients and voluntary controls underwent ambulatory overnight sleep apnea recordings (Embletta, Embla systems, Kanata, Canada) using nasal cannula pressure, thoracic and abdominal respiratory effort, finger oximetry (Nonin Oximeter, Plymouth) and a body-positioning sensor. All the recordings were scored manually according to the American Academy of Sleep Medicine recommendations. The definition of an apnea was $a \geq 90 \%$ cessation of airflow lasting at least $10 \mathrm{~s}$, while a hypopnea was defined as $50 \%$ reduction in airflow compared with baseline, in combination with an oxygen desaturation of $\geq 3 \%$ [24].

\section{Swallowing examination}

Swallowing function was investigated in all patients and voluntary controls using a videoradiographic examination (C-arm, Philips BV 29, field width $23 \mathrm{~cm}$ ) in an upright position and with lateral and frontal projections. The subjects first swallowed a chewed solid bolus of crisp bread and barium sulphate (Mixobar Esophagus; Astra) and then a liquid barium sulphate contrast bolus (Mixobar High Density; Astra). All standard boluses were repeated twice in each projection. The examinations were evaluated at full speed and slow motion by two investigators blinded for the clinical findings of the subjects. Swallowing function was graded as 1 . normal function, 2. mild dysfunction in the presence of one of the following deviant features; premature leakage, velar dysfunction, residual or laryngeal penetration, 3. moderate dysfunction with two or more deviant features in grade 2 or dysfunction of the upper esophageal sphincter, the epiglottis or the propagation wave and, 4 . severe dysfunction with aspiration below the vocal cords [25].

\section{Tissue samples and immunohistochemistry}

In patients, the entire base of the uvula was resected in connection with soft-palate surgery. In 3 of the cases, parts of the palatopharyngeus muscle were also available. The samples from voluntary controls were acquired from the corresponding site by using punch biopsy technique, except in one case where complete surgical resection of the uvula was performed. Since a punch biopsy represents only a part of the muscle cross-section, autopsies were acquired from the entire base of uvula from 5 subjects who died accidentally ( 2 males and 3 females), mean age 54 years (range 46-75), mean BMI $25 \mathrm{~kg} / \mathrm{m}^{2}$ (range 21-31). The autopsies were used only as a cross-reference to determine that the punch biopsies were representative for the entire muscle cross-section. The exclusion criteria were similar to those of voluntary controls. No medical history of snoring and sleep apnea were reported, and all subjects had a normal craniofacial and oro-pharyngeal anatomy. All samples were taken within $12-24 \mathrm{~h}$ post-mortem, a delay that not affects muscle morphology, muscle proteome and fibre typing $[26,27]$.

The muscle samples were cut into small pieces and oriented for both cross- and longitudinal sectioning. Some samples were immediately mounted in OCT (optimum cutting temperature) compound (Tissue Tek, Miles, 
Elkhart, IN, USA) and frozen in liquid propane chilled with liquid nitrogen, while others were fixed before freezing using $4 \%$ formaldehyde in $0.1 \mathrm{M}$ phosphate buffer, $\mathrm{pH}$ 7.0, for $24 \mathrm{~h}$ at $4{ }^{\circ} \mathrm{C}$ and overnight washing at $4{ }^{\circ} \mathrm{C}$ in Tyrodes solution containing $10 \%$ sucrose. Five $\mu \mathrm{m}$ thick serial muscle sections were cut in a cryostat and mounted on glass slides. The sections were immunostained with previously characterized monoclonal $(\mathrm{mAb})$ and polyclonal (pAb) antibodies directed against IF desmin, alpha-actinin (a major component of the Z-disk), membrane-associated proteins dystrophin, utrophin, and laminin, and isoforms of contractile myosin heavy chain $(\mathrm{MyHC})$ motor proteins were used (Table 1). A mounting medium with DAPI (4', 6-diamidino-2-phenylindole dihydrochloride) was used to visualize nuclei (H-1500, Vector Lab, Burlingame, CA, USA). Details of the used multi-staining technique have been described previously [28].

\section{Muscle fibre type classification}

Based on the immunostaining pattern for the different MyHC mAbs, the muscle fibres were classified as slow contracting MyHC-I (type I) or fast contracting MyHC-II (type II) fibres.

\section{Quantitative and statistical analysis}

Muscle cross-sections from uvula of all the patients and controls were included in the quantification. Palatopharyngeus muscles were not included in the quantitative analysis, due to the small sample size and lack of control biopsies. In uvula, 4 to 5 random areas from each muscle cross-section were scanned at $20 \mathrm{x}$ magnification with a fluorescence microscope (Leica DM6000B, Leica Microsystems CMS GmbH, Wetzlar, Germany) equipped with a digital high-speed fluorescence charge-coupled device
(CCD) camera (Leica DFC360 FX). The number of unstained, weakly stained, or disorderly stained fibres for the Abs directed against desmin and dystrophin were quantified manually on each photo (Photoshop CS5, version 12.0.4, San Jose, CA, USA). The investigators were blinded to the origin of the samples. Comparisons between the mean values of changes in muscle fibres between the two groups were made using the Mann-Whitney $\mathrm{U}$ test. For comparing more than two groups, planned one-way ANOVA (analysis of variance) with bootstrapping was used. Both statistical tests do not require an assumption of normality in the distribution of data. Values are presented as the mean \pm standard deviation. Results were considered significant at a $p$-value of $\leq 0.05$. All the tests were performed with SPSS (statistical package for social sciences) software (IBM SPSS 23, statistical software, Armok, NY: IBM corp., USA).

\section{Results}

All 22 patients snored, and 14 had obstructive sleep apnea (mean AHI 24, range 5-84). Ten patients had a moderate swallowing dysfunction, 6 patients had a mild dysfunction and 6 had a normal function. None of the 10 voluntary controls snored or had sleep apnea and they all displayed a normal swallowing function.

\section{Muscle morphology}

The muscle morphology of patients differed distinctly from that of the controls, by having considerably larger variability in muscle fibre size and fibre form, more loosely packed fibres, and a greater amount of connective and fat tissue (Fig. 1).

Table 1 Antibodies used for immunohistochemistry

\begin{tabular}{|c|c|c|c|c|c|c|}
\hline Antibody & Product Code & Specificity & Gene $^{a}$ & Host/Clone & Dilution & Source \\
\hline Desmin & M0760 & Human and animal desmin & DES & mAb-mouse/ D33 & $1: 100$ & 1 \\
\hline Desmin & $18-0016$ & Human desmin & DES & mAb-mouse/ ZC18 & $1: 1000$ & 2 \\
\hline Desmin & ab15200 & Human and animal desmin & DES & pAb-rabbit & $1: 2000$ & 3 \\
\hline Dystrophin & GTX15277 & Human dystrophin (C-terminus) & DMD & pAb-rabbit & $1: 7500$ & 4 \\
\hline Dystrophin & NCL-DYS1 & Human dystrophin (Rod domain) & DMD & mAb-mouse/ Dy4/6D3 & 1:5 & 5 \\
\hline Dystrophin & NCL-DYS2 & Human dystrophin (C-terminus) & DMD & mAb-mouse/ Dy8/6C5 & $1: 10$ & 5 \\
\hline Dystrophin & NCL-DYS3 & Human dystrophin ( $\mathrm{N}$ terminus) & DMD & mAb-mouse/ DY10/12B2 & $1: 10$ & 5 \\
\hline Alpha-actinin & A7732 & Human and animal a-actinin (Sarcomeric) & ACTN2 & mAb-mouse/ EA-53 & $1: 500$ & 6 \\
\hline Laminin & PC 128 & Human laminin & LAM & pAb-sheep & $1: 15000$ & 7 \\
\hline Utrophin & sc- -33700 & Human utrophin & UTRN & mAb-mouse/ 8A4 & $1: 200$ & 8 \\
\hline Slow MyHC & A4.840 & Human and animal $\mathrm{MyHCl}$ & $\mathrm{MyH} 7$ & mAb-mouse & 1:400 & 9 \\
\hline Fast A MyHC & A4.74 & Human and animal MyHClla & $\mathrm{MyH} 2$ & mAb-mouse & $1: 500$ & 9 \\
\hline
\end{tabular}

${ }^{a}$ Official gene nomenclature according to OMIM. (http://www.ncbi.nlm.nih.gov/omim). 1. Dako, Sweden, 2. Invitrogen Corporation, CA, USA; 3. Abcam, UK, 4. GeneTex Inc., Taiwan, 5. Novocastra Laboratories Ltd., UK, 6. Sigma-Aldrich Co, UK; 7. Binding site Inc., USA; 8. Santa Cruz Biotechnology Inc. UK, 9. Developmental Studies Hybridoma Bank, developed under the auspices of the NICHD and maintained by the University of Biological Sciences, lowa City, lowa, USA 


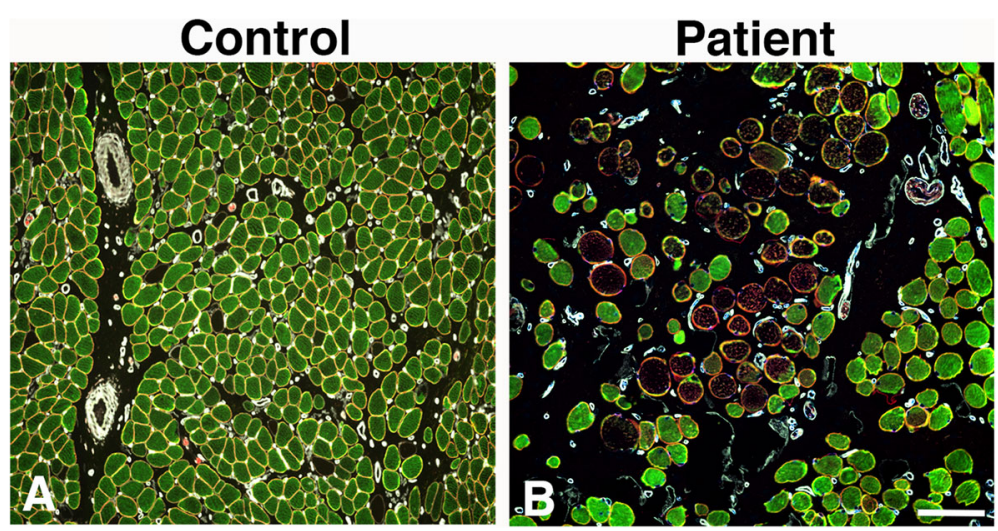

Fig. 1 Overview of muscle cross-sections from a control (a) and a patient (b) immunostained for desmin, dystrophin, and laminin (Desmin, green color, dystrophin, red color, laminin, white color, merged dystrophin and laminin, yellow color). Note the high fibre size variability, high amount of connective tissue and a large number of fibres unstained for desmin in the patient (b) compared to the control (a). Scale bar $100 \mu \mathrm{m}$

Immunoreactivity for cytoskeletal and membrane proteins Two major abnormal immunostaining patterns for desmin were observed in both small and large sized fibers in uvula and palatopharyngeus muscle cross-sections of patients. One of the patterns, observed both in controls and patients, displayed lack of immunoreactivity for desmin, here referred to as desmin-negative fibres (Figs. 1, 2, 3). The other immunoreaction pattern, exclusively present in muscle fibres of patients, displayed a disorganization of desmin ranging from fibres expressing small to large aggregates to fibres with an extensive derangement of desmin in a lobulated or trabecular pattern (Fig. 3). These fibres are referred to as desmin-disorganized fibres. A weak to intense subsarcolemmal staining for desmin was often present in both desmin-negative and desmin-disorganized fibres (Figs. 2 and 3).

In longitudinal muscle sections of patients, some of the muscle fibres showed disruptions in the normal striations of desmin in the Z-band region of the sarcomere, while others displayed complete disorganization or absence of desmin (Fig. 3 e-h). The disorganization or absence of desmin was in some fibres only located in specific regions along the length of the myofibril. Aggregates of desmin spanning over several Z-disks were often observed within the myofibrils (Fig. 3 e-h). In controls, all muscle fibres expressing desmin exhibited a normal distribution of desmin along the Z-disk, seen as horizontal striations of labeling (Fig. 3a). The immunoreaction patterns observed in both patients and controls were confirmed with all Abs directed against desmin (Table 1 ).

In control sections where desmin was co-labeled with alpha-actinin, a unique marker for the Z-disc, an intact sarcomeric structure were observed in all muscles, irrespectively of absence or presence of immunoreaction for desmin. In patients, the immunoreaction for alphaactinin in desmin negative fibres was generally weak, while all desmin disorganized fibres showed deranged immunolabeling for alpha-actinin ranging from weak to strong (Fig. 4). Muscle fibres in patients expressing a normal staining pattern for desmin also showed a normal staining pattern for alpha-actinin at the Z-disk.

The immunostaining for the different subunits of the dystrophin molecule revealed that a subgroup of muscle fibres, especially in patients, was unstained or weakly stained for the dystrophin $\mathrm{C}$-terminus $(-\mathrm{COOH})$, while the rod and the N-terminus $\left(-\mathrm{NH}_{2}\right)$ domain of the dystrophin molecule showed normal immunoreactivity in the sarcolemma (Figs. 1 and 2). All muscle fibres lacking immunoreaction for dystrophin C-terminus also displayed abnormal immunoreaction for desmin (Figs. 1 and 2). The cell membrane of all muscle fibres was normally stained for laminin in both patients and controls (Fig. 1). Utrophin, a paralog to dystrophin, was not upregulated in any muscle fibres.

All reference samples from limb muscles showed normal immunoreaction pattern for all antibodies directed against cytoskeletal and membrane proteins.

\section{Quantification of cytoskeletal abnormalities}

The quantification and statistical analyses of fibres with abnormal expression of desmin and dystrophin in the uvula muscle were based on an evaluation of 7836 muscle fibres in patients and 1443 muscle fibres in the biopsies from voluntary controls.

For cross-reference, the biopsies from voluntary controls and patients were compared with the results from control autopsies ( $n=3453$ muscle fibres).

\section{Proportion of desmin and dystrophin abnormalities}

All samples from patients had cytoskeletal abnormalities in the muscle fibre population. The proportion of desmin-disorganized fibres was $21 \pm 13 \%$ in patients, while they were not present in controls (Fig. 3 i). No specific fibre type predilection was observed for fibres with disorganized desmin. Desmin-negative fibres were 

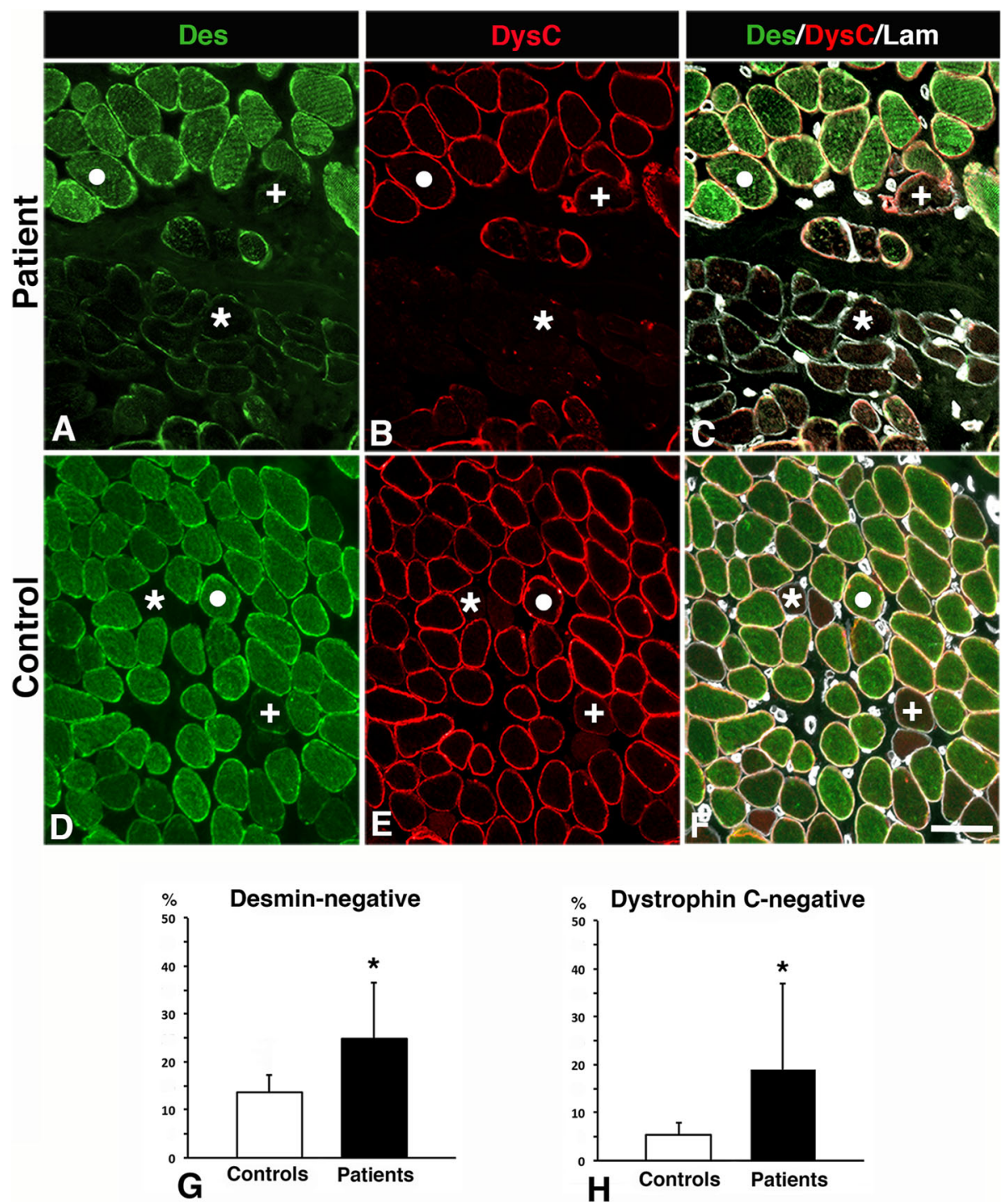

Fig. 2 Muscle cross-sections from a patient (a-c) and a control (d-f) immunostained for desmin (Des, green colour, a, d), dystrophin C-terminus (DysC, red colour, $\mathbf{b}, \mathbf{e}$ ) and laminin (Lam, white colour, c, f). Panels C and F show merged staining. Normal expression of desmin and dystrophin (white dot), desmin-negative fibres (+) and desmin and dystrophin C-terminus-negative fibres $\left({ }^{*}\right)$. Scale bar $50 \mu \mathrm{m}$. Graphs $\mathbf{g}$ and $\mathbf{h}$ shows the percentage of desmin and dystrophin C-terminus negative fibres in controls and patients (mean and SD). A significant difference $(p<0.05)$ is marked $\left(^{*}\right)$

found in $25 \pm 12 \%$ of the fibre population in patients, which was significantly more than in controls $(14 \pm 7 \%, p$ $=0.009$ ) (Fig. 2 g). These fibres were predominantly of slow type I $(64 \pm 22 \%)$ (Fig. 5). The pooled fractions of desmin-negative and desmin-disorganized fibres (46 \pm $18 \%$ ), referred to as desmin-abnormal fibres, were significantly higher in patients than in controls $(p<0.001)$ (Fig. $3 \mathrm{j})$. The frequency of dystrophin $\mathrm{C}$-terminus-negative fibres was also significantly higher in patients $(19 \pm 18 \%)$ compared to controls $(7 \pm 2 \%, p=0.04)$ (Fig. $2 \mathrm{~h}$ ).

\section{Cross-reference with control autopsies}

No significant differences in muscle morphology and proportions of desmin-negative fibres were observed between control autopsies and control biopsies. Desmin disorganized fibres were not observed either in control biopsies or autopsies. The proportion of cytoskeletal abnormalities in patient biopsies was significantly higher compared to control autopsies (data not shown).

Proportion of desmin and dystrophin abnormalities in patients with and without a swallowing dysfunction

Both desmin-negative and desmin-disorganized muscle fibres were significantly higher in patients with swallowing dysfunction compared with patients with normal function $(p=0.005)$ (Fig. 6 a and b).

The pooled fractions of desmin-negative and desmindisorganized fibres i.e. desmin-abnormal fibres, were 55 

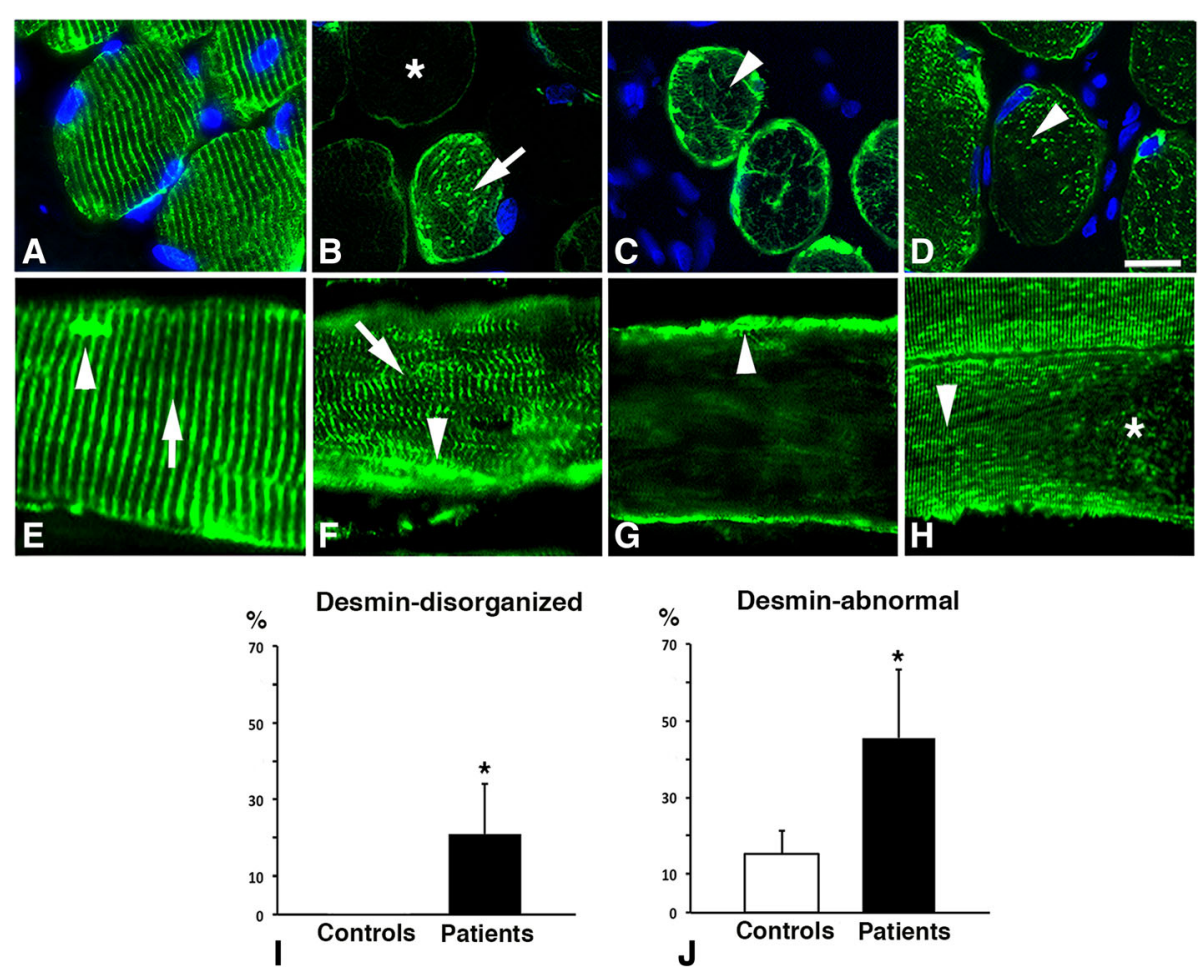

Fig. 3 Muscle cross-sections (a-d) and longitudinal sections (e-h) stained for desmin and nuclei (DAPI) in the uvula muscle of patients. Normal immunoreaction for desmin is shown in (a) and abnormal immunoreaction patterns are shown in (b-h). Panel $\mathbf{b}$ shows a desminnegative fibre $\left(^{*}\right)$ and a fibre with desmin distributed as larger dots or striations (arrow). Panel c displays an irregular or trabecular pattern of desmin (arrowhead) and panel $\mathbf{d}$ shows desmin evenly distributed as fine grainy dots (arrowhead). Panel e shows a longitudinally sectioned muscle fibre with a normal striated pattern of desmin in the Z-line (arrow) and subsarcolemmal aggregation of desmin (arrowhead). Panel $\mathbf{f}$ displays myofibrillar disorganization (arrow) and desmin aggregates (arrowhead), Panel $\mathbf{g}$ shows a desmin-negative fibre with dense subsarcolemmal staining (arrowhead) and panel $\mathbf{h}$ shows an area with a striated and punctuated staining pattern for desmin (arrowhead) and an area lacking desmin $\left(^{*}\right)$. Scale bar $\mathbf{a}-\mathbf{h}, 25 \mu \mathrm{m}$. Bar graphs showing the percentage of fibres with disorganized desmin (i) and the pooled values of desmin-negative and desmin-disorganized fibres (j, desmin-abnormal) in controls and patients (mean and SD). A significant difference $(p<0.05)$ is marked $(*)$

$\pm 10 \%$ in patients with a mild to moderate swallowing dysfunction compared to $22 \pm 6 \%$ in patients with a normal swallowing function, ( $p=0.002)$ (Fig. $6 \mathrm{c}$ ).

The frequency of dystrophin C-terminus-negative fibres was higher in patients with a swallowing dysfunction than in patients with a normal function (18 $\pm 13 \%$ vs. $7 \pm 5 \%, p$ $=0.007)$.

\section{Comparison between cytoskeletal abnormalities and $\mathrm{AHI}$}

All snoring and sleep apnea patients had a significantly higher proportion of cytoskeletal abnormalities compared to controls, but the degree of abnormalities did not show any significant relationship with the severity of AHI or oxygen desaturation.

\section{Discussion}

We here report evidence of cytoskeletal abnormalities in soft palate muscles of snoring and sleep apnea patients. A significant number of muscle fibres revealed an absence, derangement or an abnormal isoform of two important cytoskeletal proteins, desmin, and dystrophin. Interestingly, these cytoskeletal abnormalities were significantly more frequent in snoring and sleep apnea patients with swallowing dysfunction than in patients with normal swallowing function. The present findings highlight that apart from previous reports of sensorimotor neuropathy in upper airways [5-10,29,30], cytoskeletal abnormalities in muscles have to be taken into account in the pathophysiology of pharyngeal dysfunction in snorers and sleep apnea patients. Any changes rendering the upper-airway muscles less capable of generating force have the potential to affect pharyngeal function.

The high proportion of fibres with disorganized desmin as well as histopathological changes such as atrophic and hypertrophic fibres, fascicular atrophy and fibrosis in the palate muscles of snorer and sleep apnea reflect muscle weakness in the upper airways due to local injury. The disorganization of desmin often resembled the pattern seen in myofibrillar myopathies where the primary clinical feature is progressive muscle weakness [22, 31]. The 

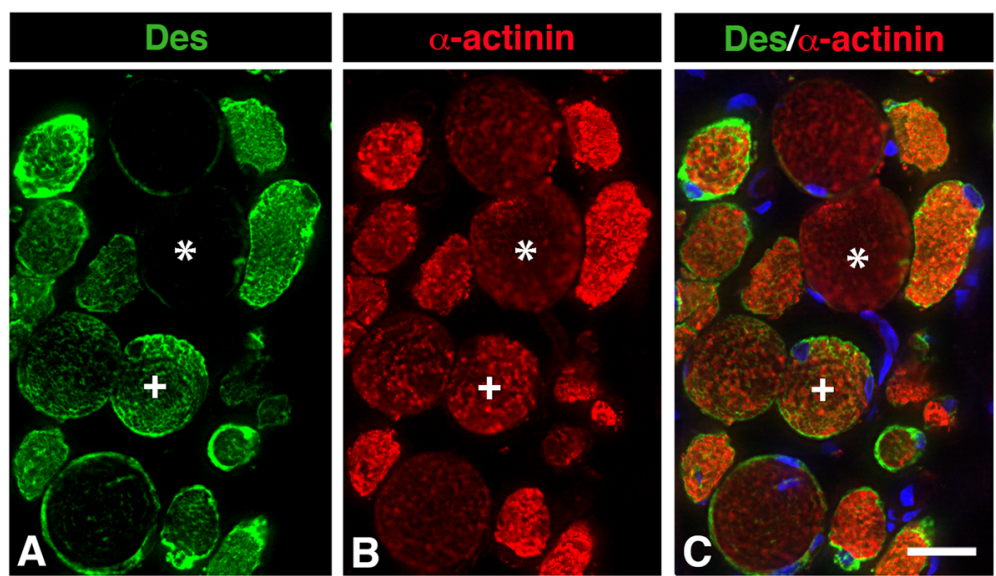

Fig. 4 Muscle cross-section from the uvula of a patient immunostained for desmin (Des, green color, a) and alpha-actinin (a-actinin, red color, b). Panels c shows merged staining for desmin, alpha-actinin, and nuclei (DAPI). A desmin negative fibre weakly stained for alpha-actinin $\left(^{*}\right)$ and a fibre with immunoreaction for both desmin and alpha-actinin (+). Note the large variability in staining pattern and intensity for both desmin and alpha-actinin. Scale bar $25 \mu \mathrm{m}$

pathogenesis of these two distinct types of changes seem, however, to differ. While the presence of atrophic fibers is probably due to acquired motor neuropathy secondary to snoring vibratory damage $[5,12-14,32]$, the desmin disorganized fibres seems preferably related to myofibrillar injury and muscle overload. This assumption is based on the fact that hypertrophic fibres also displayed disorganized desmin, indicating a frequent use and thus an intact nerve-innervation. Moreover, cytoskeletal disruption has been reported in muscle fibres subjected to overload [33, 34]. Nonetheless, denervation cannot be excluded for the changes of desmin observed in fibres of smaller size. Volodin et al., [35] reported that slow muscle fibre atrophy induced by denervation initially causes phosphorylation and ubiquitination of desmin, followed by dissociation of desmin filaments and protein degradation. However, this process may account for desmin abnormalities in fibres with an atrophic appearance, but not for the abnormalities in the hypertrophic fibres.

Another possible basis for desmin abnormalities is local disturbances in blood circulation in snorers. There is evidence that long-standing exposure to mechanical vibrations in limbs causes vascular damage, reduction in blood flow, mitochondrial disorganization, as well as cytoskeletal derangement and decreased muscle strength [36-38]. Disturbed blood circulation in limb muscles due to peripheral artery disease (PAD) has also been shown to cause an abnormal distribution of desmin as well as an aberrant muscle fibre morphology including irregular and patchy distribution of the mitochondria
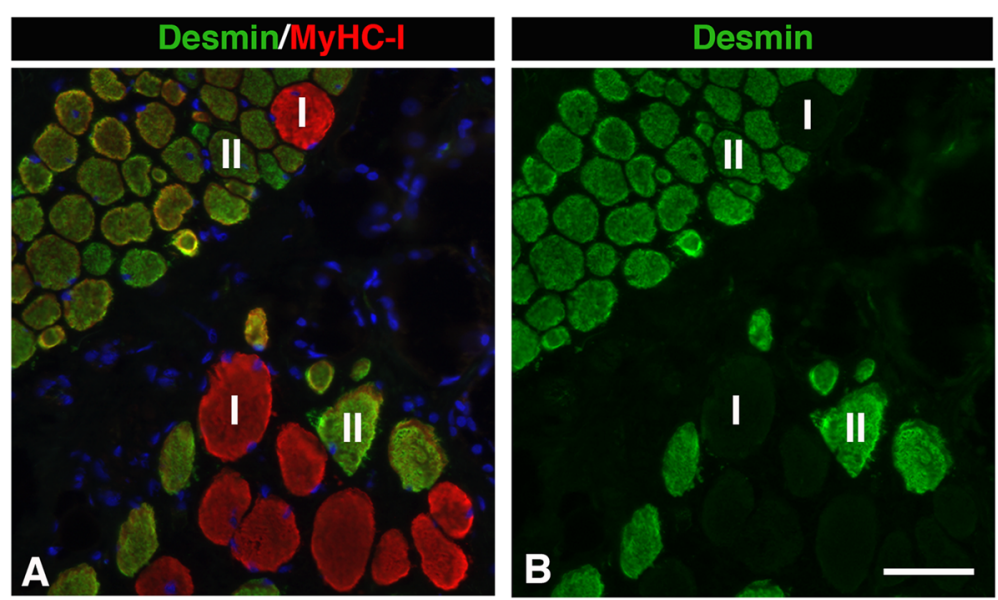

Fig. 5 Muscle cross-section from the uvula of a patient stained for desmin and slow contractile protein MyHCl. Panel a shows merged staining for desmin and slow $\mathrm{MyHCl}$, while panel $\mathbf{b}$ shows only desmin. Fibres expressing slow $\mathrm{MyHCl}($ red, I) and fast MyHC II (green, II) are marked. Note that the deficiency of desmin is more common among fibres expressing MyHCl. Scale bar $50 \mu \mathrm{m}$ 

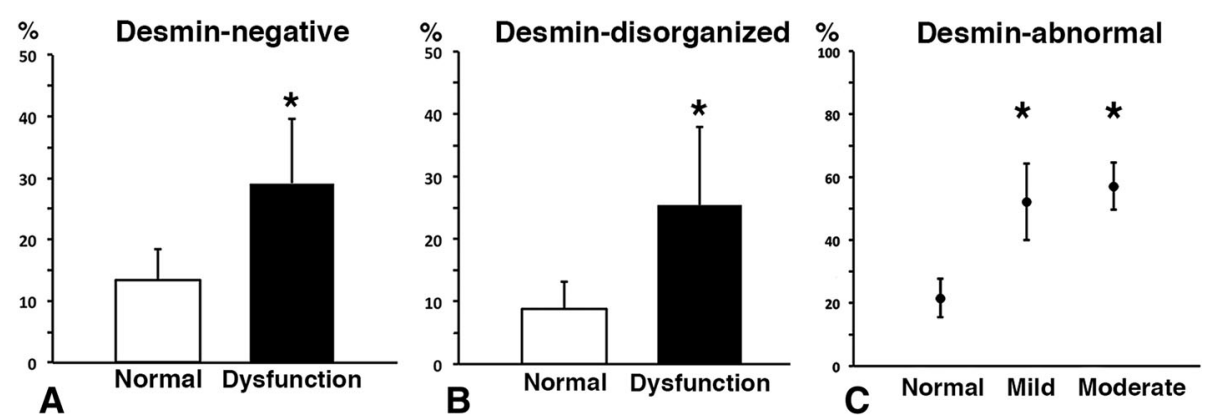

Fig. 6 Bar graphs showing the percentage of desmin-negative fibres (a) and desmin-disorganized fibres (b) in the uvula muscle of patients with normal swallowing or dysfunctional swallowing (mean and SD). Graph (c) shows proportion (\%) of desmin-abnormal fibres, i.e. pooled percentage of desmin-negative and desmin-disorganized fibres, in patients with normal swallowing function and mild and moderate swallowing dysfunction, respectively (mean and SD). A significant difference $(p<0.05)$ is marked $\left(^{*}\right)$

[39]. Interestingly, this maldistribution of desmin and mitochondria resembles the abnormal derangement of desmin and mitochondrial distribution previously reported in palate muscle fibres of patients [40]. Desmin has a key role in anchoring of mitochondria, and mutations of desmin are reported to substantially disturb spatial orientation and function of mitochondria in muscles $[18,19]$. Accordingly, disorganization of desmin and mitochondria, together with our previous findings of reduced muscle capillarization in the soft palate of long-term snorers [41], underpins a disturbed energy production and muscle weakness.

The novel finding of muscle fibres lacking desmin in the soft palate of both patients as well as healthy adult and infants [15], raises interesting questions about their origin. Muscle fibres containing disorganized or disrupted desmin have been described in several myopathies [31], but no disease or neuromuscular injuries have been related to lack of desmin in muscle fibres. Although a transient loss of desmin has been observed in muscles following acute or eccentric activity in animal experiments, the expression of desmin returned to normal levels after a short time [33, 34]. Interestingly, Janbaz et al. [42] reported that a subgroup of fibres in healthy human extraocular muscles lacked or had faint staining for desmin. Extraocular muscles have, as palate muscles, intricate muscle anatomy with several muscles lacking firm attachment at one end and acting against each other in performing precise movements of the eye. In light of this, the presence of fibres lacking desmin can constitute an evolutionary cytoskeletal specialization of the muscle fibres to meet requirements in various oropharyngeal functions. Additionally, while desmin disorganized fibres did not have any fibre type predilection, fibres lacking desmin were mainly of slow phenotype I, a finding further strengthening cytoskeletal fibre phenotype specialization. Thus, the significantly higher proportion of fibres with an absence of desmin in muscles from patients compared to healthy controls indicates either a genetic link or that these fibres better survive muscle overload or neuromuscular trauma by snoring and tissue stretch. Since fibres lacking desmin preferentially were of slow type I, the high proportion of desmin negative fibres in snoring and sleep apnea patients could relate to the fact that the motor-nerve damage preferentially affects fibres belonging to type II motor-units, which is in line with some previous studies $[7,12]$. The reason why cytoskeletal abnormalities in certain fibres were only observed in specific regions along the length of myofibril needs further investigation.

Another significant finding was that a subpopulation of the desmin-abnormal fibres also lacked immunoreactivity for the $\mathrm{C}$-terminus of dystrophin. The $\mathrm{C}$-terminus domain of the dystrophin molecule is considered to be crucial for normal muscle function, as it binds to the glycoprotein complex in the sarcolemma and transfers forces from the contractile apparatus to the extracellular matrix and adjoining muscle fibres [20, 23]. Physiological studies demonstrated that force production is significantly reduced in muscle fibres showing a deficiency of dystrophin [43]. Moreover, extensive data report that a dystrophin-deficient sarcolemma is fragile and results in increased permeability of membrane-impermeable molecules, especially after physical exercise. In Duchenne muscular dystrophy, loss or truncated forms of dystrophin leave the membrane highly susceptible to contraction-induced injury and hypoxic stress [44], which has deleterious consequences for the intra-myofibrillar environment. Thus, the high proportion of dystrophin $\mathrm{C}$-terminus deficient fibres in snorers and sleep apnea patients might render the muscle more vulnerable to high contraction stress.

Utrophin, a paralog to dystrophin, is expressed on the sarcolemma of developing and regenerating fibres, but it is ultimately replaced by dystrophin in maturing fibres [45]. In Duchenne muscular dystrophy, where dystrophin 
is lacking, utrophin is strikingly upregulated [46]. However, utrophin was not upregulated in muscle fibres lacking immunoexpression for the dystrophin C-terminus in both patients and controls. Hence, further studies have to rule out whether the truncated form of dystrophin in desmin abnormal fibres is a consequence of local injuries or that the C-terminal of the dystrophin molecule has a specific configuration in soft palate muscles.

Several investigators have proposed that obstructive sleep apnea is a progressive heavy snorers disease [47-49]. Our findings reinforce traumatic snoring vibrations as well as muscle overload as the most credible mechanism for the high presence of fibres with abnormal cytoskeletal distribution in patients. This is strengthened by the observation that alpha-actinin was weakly stained or deranged in most of the desmin abnormal fibres in patients, while all fibres in controls, irrespectively of desmin expression, showed an intact $\mathrm{Z}$ disk structure. Additionally, the findings that long-standing exposure to vibrations causes nerve and muscle injuries, including cytoskeletal and mitochondrial disorganization, vascular damage, reduction in blood flow, and decreased muscle strength further support vibratory trauma as a cause for structural muscle changes. Moreover, contraction during lengthening of muscle has been reported to cause histopathological changes in muscle fibres [34], a process that can occur during sleep when the dilator muscles try to counteract the collapsing forces while breathing. Therefore, nerve and muscle injuries, as well as disturbances in blood flow, may affect pharyngeal function in snorers that over time contributes to the development of sleep apnea. Treatment strategies aimed at reducing trauma from snoring and muscle overload, as well as strengthening the upper-airway muscles, could have long-term benefits.

Although the proportion of desmin and dystrophin abnormalities in sleep apnea patients were significantly higher than in controls, no relation to the severity of sleep apnea (AHI) could be established. This reflects the complex interaction of several central and peripheral etiological risk factors in the development and progression of obstructive sleep apnea [50]. Hence, the impact of cytoskeletal abnormalities on upper airway muscle function should be considered together with factors such as the degree of muscle relaxation during sleep, upper airway anatomy, nerve injuries, and other co-morbid conditions in snorers and sleep apnea patients. Future studies with larger cohorts could shed more light on the question if a particular genotype or phenotype of patients exists based on the present findings in snorers and sleep apnea patients.

\section{Conclusions}

To conclude, the present data show that the expression of cytoskeletal proteins in muscle fibres of the soft palate in snoring and sleep apnea patients differ from healthy individuals and from limb muscles. Cytoskeletal abnormalities including absence, disorganization, and accumulation of desmin as well as a truncated form of dystrophin were common in the soft palate muscles of patients, but not in controls. The link between cytoskeletal abnormalities and swallowing dysfunction supports impaired muscle function in the upper airways of patients. Hence, in addition to neuropathy, cytoskeletal myopathy seems to be a contributing factor in the pathophysiology of pharyngeal muscle dysfunction in the upper airways of sleep apnea patients.

\section{Abbreviations \\ AHI: Apnea-hypopnea index; ANOVA: Analysis of variance; BMI: Body mass index; CCD: Charged couple device; DAPI: 4', 6-diamidino-2-phenylindole dihydrochloride; mAb: Monoclonal antibody; MyHC: Myosin heavy chain; OCT: Optimum cutting temperature; pAb: Polyclonal antibody; \\ PAD: Peripheral artery disease; SD: Standard deviation; SPSS: Statistical package for social sciences}

\section{Acknowledgments}

We thank Mrs. Anna-Karin Olofsson (Department of Integrative Medical Biology, Laboratory of Muscle Biology, Umeå University, Umeå, Sweden) for her excellent technical assistance.

\section{Funding}

Swedish Heart and Lung Foundation (Dnr 20110210, 20140339), Swedish Research Council (Dnr 2018-02574), the JC Kempe Memorial Scholarship Foundation, the Swedish Dental Society and Insamlingsstiftelsen Umeå University (Dnr 223-1881-13).

\section{Availability of data and materials}

Any data generated and/or analysed during the current study are available from the corresponding author on reasonable request.

\section{Authors' contributions}

PS is the guarantor of the study. DB, EL, and PS conceived the study. FS, EL, $D B$, and PS designed the study. FS, KF, SF, TH, ELJ, and PS did acquisition and analysis of the data. FS and PS conducted the statistical analysis. All authors contributed to the interpretation of the findings. FS and PS prepared the draft of the manuscript and all authors critically revised the manuscript for intellectually important content. All authors gave final approval for the publication of the work, and all accepted responsibility for the integrity of the work.

\section{Ethics approval and consent to participate}

The regional Medical Ethical Committee in Umeå approved the study (Dnr05-130 M). The patients and voluntary controls were informed and gave their written consent to participate. All muscle samples were collected in agreement with the Declaration of Helsinki. Autopsy specimens were collected in agreement with Swedish laws and regulations on autopsy and transplantation.

\section{Consent for publication}

Not applicable.

\section{Competing interests}

The authors declare that they have no competing interests.

\section{Publisher's Note}

Springer Nature remains neutral with regard to jurisdictional claims in published maps and institutional affiliations.

\section{Author details}

${ }^{1}$ Department of Integrative Medical Biology, Laboratory of Muscle Biology, Umeå University, SE-901 87 Umeå, Sweden. ²Department of Surgical and Perioperative Sciences, Surgery, Umeå University, Umeå, Sweden. 
${ }^{3}$ Department of Clinical Sciences, Otolaryngology, Umeå University, Umeå, Sweden. ${ }^{4}$ Department of Odontology, Oral and Maxillofacial Radiology, Umeå University, Umeå, Sweden.

Received: 30 October 2018 Accepted: 4 February 2019

Published online: 14 February 2019

\section{References}

1. Franklin KA, Lindberg E. Obstructive sleep apnea is a common disorder in the population-a review on the epidemiology of sleep apnea. J Thorac Dis. 2015:7:1311-22. https://doi.org/10.3978/j.issn.2072-1439.2015.1306.1311.

2. Dempsey JA, Veasey SC, Morgan BJ, O'Donnell CP. Pathophysiology of sleep apnea. Physiol Rev. 2010;90:47-112. https://doi.org/10.1152/physrev.00043. 02008.

3. Jäghagen EL, Berggren D, Isberg A. Swallowing dysfunction related to snoring: a videoradiographic study. Acta Otolaryngol. 2000;120:438-43.

4. Levring Jäghagen $E$, Franklin KA, Isberg A. Snoring, sleep apnoea and swallowing dysfunction: a videoradiographic study. Dentomaxillofac Radiol. 2003:32:311-6

5. Shah F, Holmlund T, Jäghagen EL, Berggren D, Franklin K, Forsgren S, Stal P. Axon and Schwann cell degeneration in nerves of upper airway relates to pharyngeal dysfunction in snorers and patients with sleep apnea. Chest. 2018;30:30963-2

6. Boyd JH, Petrof BJ, Hamid Q, Fraser R, Kimoff RJ. Upper airway muscle inflammation and denervation changes in obstructive sleep apnea. Am J Respir Crit Care Med. 2004;170:541-6 Epub 2004 May 2019.

7. Friberg D, Ansved T, Borg K, Carlsson-Nordlander B, Larsson H, Svanborg E. Histological indications of a progressive snorers disease in an upper airway muscle. Am J Respir Crit Care Med. 1998;157:586-93.

8. Hagander L, Harlid R, Svanborg E. Quantitative sensory testing in the oropharynx: a means of showing nervous lesions in patients with obstructive sleep apnea and snoring. Chest. 2009;136:481-9. https://doi.org/ 10.1378/chest.1308--2747 Epub 2009 Jun 1319.

9. Podnar S, Dolenc Groselj L. Neuropathic changes in the tongue protruder muscles in patients with snoring or obstructive sleep apnea. Neurophysio Clin. 2018;48:269-75. https://doi.org/10.1016/j.neucli.2018.1005.1040 Epub 2018 Jun 1015.

10. Saboisky JP, Stashuk DW, Hamilton-Wright A, Carusona AL, Campana LM, Trinder J, Eckert DJ, Jordan AS, McSharry DG, White DP, et al. Neurogenic changes in the upper airway of patients with obstructive sleep apnea. Am J Respir Crit Care Med. 2012;185:322-9. https://doi.org/10.1164/rccm.2011062010580C Epub 202011 Oct 201120.

11. Kimoff RJ. Upper airway myopathy is important in the pathophysiology of obstructive sleep apnea. J Clin Sleep Med. 2007;3:567-9.

12. Lindman R, Stål PS. Abnormal palatopharyngeal muscle morphology in sleep-disordered breathing. J Neurol Sci. 2002;195:11-23.

13. Edström L, Larsson H, Larsson L. Neurogenic effects on the palatopharyngeal muscle in patients with obstructive sleep apnoea: a muscle biopsy study. J Neurol Neurosurg Psychiatry. 1992;55:916-20.

14. Woodson BT, Garancis JC, Toohill RJ. Histopathologic changes in snoring and obstructive sleep apnea syndrome. Laryngoscope. 1991;101:1318-22.

15. Shah F, Berggren D, Holmlund T, Levring Jäghagen E, Stål P. Unique expression of cytoskeletal proteins in human soft palate muscles. J Anat. 2016:228:487-94 10.1111/joa.12417. Epub 12015 Nov 12424

16. Paulin D, Li Z. Desmin: a major intermediate filament protein essential for the structural integrity and function of muscle. Exp Cell Res. 2004;301:1-7.

17. Small JV, Furst DO, Thornell LE. The cytoskeletal lattice of muscle cells. Eur J Biochem. 1992;208:559-72

18. Milner DJ, Mavroidis M, Weisleder N, Capetanaki Y. Desmin cytoskeleton linked to muscle mitochondrial distribution and respiratory function. J Cell Biol. 2000:150:1283-98

19. Winter L, Wittig I, Peeva V, Eggers B, Heidler J, Chevessier F, Kley RA, Barkovits K, Strecker V, Berwanger C, et al. Mutant desmin substantially perturbs mitochondrial morphology, function and maintenance in skeletal muscle tissue. Acta Neuropathol. 2016:132:453-73.

20. Campbell KP. Three muscular dystrophies: loss of cytoskeleton-extracellular matrix linkage. Cell. 1995;80:675-9.

21. Petrof BJ, Shrager JB, Stedman HH, Kelly AM, Sweeney HL. Dystrophin protects the sarcolemma from stresses developed during muscle contraction. Proc Natl Acad Sci U S A. 1993;90:3710-4.
22. Clemen CS, Herrmann H, Strelkov SV, Schröder R. Desminopathies: pathology and mechanisms. Acta Neuropathol. 2013;125:47-75. https://doi. org/10.1007/s00401-00012-01057-00406 Epub 02012 Nov 00411.

23. Rahimov F, Kunkel LM. The cell biology of disease: cellular and molecular mechanisms underlying muscular dystrophy. J Cell Biol. 2013;201:499-510. https://doi.org/10.1083/jcb.201212142

24. Berry RB, Budhiraja R, Gottlieb DJ, Gozal D, Iber C, Kapur VK, Marcus CL, Mehra R, Parthasarathy S, Quan SF, et al. Rules for scoring respiratory events in sleep: update of the 2007 AASM Manual for the Scoring of Sleep and Associated Events. Deliberations of the Sleep Apnea Definitions Task Force of the American Academy of Sleep Medicine. J Clin Sleep Med. 2012;8:597619 10.5664/jcsm.2172.

25. Jäghagen $\mathrm{EL}$, Bodin I, Isberg A. Pharyngeal swallowing dysfunction following treatment for oral and pharyngeal cancer--association with diminished intraoral sensation and discrimination ability. Head Neck. 2008; 30:1344-51 10.1002/hed.20881

26. Tavichakorntrakool R, Prasongwattana V, Sriboonlue P, Puapairoj A, Pongsku J, Khuntikeo N, Hanpanich W, Yenchitsomanus PT, Wongkham C, Thongboonkerd $\mathrm{V}$. Serial analyses of postmortem changes in human skeletal muscle: A case study of alterations in proteome profile, histology, electrolyte contents, water composition, and enzyme activity. Proteomics Clin Appl. 2008;2:1255-64. https://doi.org/10.1002/prca.200800051 Epub 200802008 Jul 200800030.

27. Eriksson O, Eriksson A, Ringqvist M, Thornell LE. The reliability of histochemical fibre typing of human necropsy muscles. Histochemistry. 1980;65:193-205.

28. Lindström M, Thornell LE. New multiple labelling method for improved satellite cell identification in human muscle: application to a cohort of power-lifters and sedentary men. Histochem Cell Biol. 2009;132:141-57. https://doi.org/10.1007/s00418--00009--00606-00410 Epub 02009 May 00431.

29. Svanborg E. Upper airway nerve lesions in obstructive sleep apnea. Am J Respir Crit Care Med. 2001;164:187-9. https://doi.org/10.1164/ajrccm.1164. 1162.2105010c.

30. Sunnergren O, Brostrom A, Svanborg E. Soft palate sensory neuropathy in the pathogenesis of obstructive sleep apnea. Laryngoscope. 2011;121:451-6 doi: 10.1002/lary.21371

31. Carlsson L, Thornell LE. Desmin-related myopathies in mice and man. Acta Physiol Scand. 2001;171:341-8. https://doi.org/10.1046/j.1365-1201x.2001. 00837.x.

32. Shah FFS, Holmlund $T$, Jäghagen EL, Berggren D, Franklin $K$, Stål $P$. Neurotrophic factor BDNF is upregulated in soft palate muscles of snorers and sleep apnea patients. Laryngoscope Invest Otolaryngol. 2019. https:// onlinelibrary.wiley.com/doi/full/10.1002/lio2.225.

33. Barash IA, Peters D, Friden J, Lutz GJ, Lieber RL. Desmin cytoskeletal modifications after a bout of eccentric exercise in the rat. Am J Physiol Regul Integr Comp Physiol. 2002;283:R958-63.

34. Lieber RL, Thornell LE, Friden J. Muscle cytoskeletal disruption occurs within the first 15 min of cyclic eccentric contraction. J Appl Physiol (1985). 1996; 80:278-84.

35. Volodin A, Kosti I, Goldberg AL, Cohen S. Myofibril breakdown during atrophy is a delayed response requiring the transcription factor PAX4 and desmin depolymerization. Proc Natl Acad Sci U S A. 2017;114:E1375-84. https://doi.org/10.1073/pnas.1612988114 Epub 1612982017 Jan 1612988117.

36. Murfee WL, Hammett LA, Evans C, Xie L, Squire M, Rubin C, Judex S, Skalak TC. High-frequency, low-magnitude vibrations suppress the number of blood vessels per muscle fiber in mouse soleus muscle. J Appl Physiol (1985). 2005:98:2376-80 Epub 2005 Jan 2327.

37. Necking LE, Lundborg G, Lundström R, Thornell LE, Friden J. Hand muscle pathology after long-term vibration exposure. J Hand Surg $\mathrm{Br}$. 2004;29:431-7.

38. Curry BD, Bain JL, Yan JG, Zhang LL, Yamaguchi M, Matloub HS, Riley DA. Vibration injury damages arterial endothelial cells. Muscle Nerve. 2002;25: 527-34.

39. Koutakis P, Miserlis D, Myers SA, Kim JK, Zhu Z, Papoutsi E, Swanson SA, Haynatzk G, Ha DM, Carpenter LA, et al. Abnormal accumulation of desmin in gastrocnemius myofibers of patients with peripheral artery disease: associations with altered myofiber morphology and density, mitochondrial dysfunction and impaired limb function. J Histochem Cytochem. 2015;63:256-69.

40. Stål PS, Johansson B. Abnormal mitochondria organization and oxidative activity in the palate muscles of long-term snorers with obstructive sleep 
apnea. Respiration. 2012;83:407-17. https://doi.org/10.1159/000336040 Epub 000332012 Mar 000336041.

41. Stål PS, Lindman R, Johansson B. Capillary supply of the soft palate muscles is reduced in long-term habitual snorers. Respiration. 2009;77:303-10. https://doi.org/10.1159/000197975 Epub 000192009 Jan 000197929.

42. Janbaz AH, Lindström M, Liu JX, Pedrosa Domellöf F. Intermediate filaments in the human extraocular muscles. Invest Ophthalmol Vis Sci. 2014;55:51519. https://doi.org/10.1167/iovs.5114-14316.

43. Deconinck N, Rafael JA, Beckers-Bleukx G, Kahn D, Deconinck AE, Davies $\mathrm{KE}$, Gillis JM. Consequences of the combined deficiency in dystrophin and utrophin on the mechanical properties and myosin composition of some limb and respiratory muscles of the mouse. Neuromuscul Disord. 1998:8:362-70.

44. Canton M, Menazza S, Di Lisa F. Oxidative stress in muscular dystrophy: from generic evidence to specific sources and targets. J Muscle Res Cell Motil. 2014;35:23-36. https://doi.org/10.1007/s10974-10014-19380-10972 Epub 12014 Mar 10912.

45. Helliwell TR, Man NT, Morris GE, Davies KE. The dystrophin-related protein, utrophin, is expressed on the sarcolemma of regenerating human skeletal muscle fibres in dystrophies and inflammatory myopathies. Neuromuscul Disord. 1992;2:177-84.

46. Hirst RC, McCullagh KJ, Davies KE. Utrophin upregulation in Duchenne muscular dystrophy. Acta Myol. 2005;24:209-16.

47. Petrof BJ, Hendricks JC, Pack Al. Does upper airway muscle injury trigger a vicious cycle in obstructive sleep apnea? A hypothesis. Sleep. 1996;19:465-71.

48. Svanborg E, Larsson H. Natural evolution of obstructive sleep apnea syndrome. Sleep. 1993;16:S124-5.

49. Lugaresi E, Plazzi G. Heavy snorer disease: from snoring to the sleep apnea syndrome--an overview. Respiration. 1997;64:11-4. https://doi.org/10.1159/ 000196730.

50. Hudgel DW. Sleep apnea severity classification - revisited. Sleep. 2016;39: 1165-6.

Ready to submit your research? Choose BMC and benefit from:

- fast, convenient online submission

- thorough peer review by experienced researchers in your field

- rapid publication on acceptance

- support for research data, including large and complex data types

- gold Open Access which fosters wider collaboration and increased citations

- maximum visibility for your research: over $100 \mathrm{M}$ website views per year

At $\mathrm{BMC}$, research is always in progress.

Learn more biomedcentral.com/submissions 